XIX.

Aus der inneren Abtheilung des städt. Luisenhospitals zu Dortmund (ehem. Chefarzt: Dr. F. Volhard, jetzt Director der städt. Krankenanstalten in Mannheim).

\title{
Ueber die Beeinflussung des systolischen und diastolischen Blutdrucks durch Tabackrauchen.
}

\author{
Von \\ Dr. med. M. John, \\ ehem. Secundärarzt der Abth., jetat Chefarzt der inneren Abth. am Marienhospital in Mulheim a. Ruhr. \\ (Hierzu Tafeln XIV—XVIII.)
}

Bereits vor einer Reihe von Jahren, als ich Untersuchungen über die Beeinflussung des Blutdruckes durch Alkohol anstellte, habe ich auch, zum Theil bei den gleichen Versuchspersonen, den Effect des Tabackrauchens auf das Gefässsystem eingehend studirt. Inzwischen ist diese Frage von Nicolai und Stähelin bearbeitet worden, die auf Grund vieler, unter Zuhülfenahme sehr feiner Untersuchungsmethoden an sich selbst durchgeführter Versuche zu dem Schlusse gelangten, „dass fortgesetztes Tabackrauchen auch dann, wenn es nicht zu eigentlichen Intoxicationserscheinungen führt, die Anspruchsfähigkeit der Gefässe schädigt". Nichtsdestoweniger glaube ich, dass meine Versuche, die an einem grösseren Material angestellt sind, noch in mancher Beziehung interessante Einzelheiten ergeben, zumal ich von den meisten Versuchspersonen ausser den gewöhnlichen nicotinhaltigen auch sogenannte nicotinarme Cigarren, ferner Cigaretten und endlich Cigarren in Mengen von 8-10 Stück, über den ganzen Tag vertheilt, rauchen liess. Derartige, nach allen diesen yichtungen hin ausgedehnte Versuche geben nicht bloss Aufschluss über die Beeinflussung des Gefässsystems durch das Rauchen, sondern klären auch die nicht ganz unwichtige Frage, ob die sogenannten nicotinarmen Cigarren den nicotinhaltigen gegenüber als unbedenklicher zu gelten haben, und ob die Cigaretten thatsächlich, wie vielfach behauptet wird, schädlicher sind als Cigarren.

Wie seiner Zeit bei den Alkoholversuchen habe ich den Effect des Rauchens auf das Gefässsystem durch fortlaufende Messungen des systolischen und diastolischen Druckes und ständige Controle des Pulses zu eruiren gesucht. Und obgleich eine Bestimmung des diastolischen Druckes nach der v. Recklinghausen'schen Methode an und für sich nicht absolut exact durchfübrbar ist, da eben die Oscillationen nur allmählich kleiner werden, und obgleich Aenderungen der Amplitude an den Armgefässen noch nicht ohne Weiteres ein getreues Spiegelbild der Vorgänge im Gesammtgefässsystem geben, babe ich doch durchaus brauchbare Messresultate erhalten. Denn bei einer ganzen Anzahl von Männern - 
und natürlich sind nur solche zu Versuchen herangezogen worden liess sich bei allen Messungen immer eine bestimmte Phase, sagen wir, in der Nähe des diastolischen Druckes, fixiren, wo am Recklinghausenschen Tonometer die maximalen Oscillationen von 5 Theilstrichbreite eben in solche von 4 Theilstrichbreite oder letztere in solche von 3 Theilstrichbreite übergehen. Ferner sind alle Eventualitäten, die Einfluss auf die Druckverhältnisse im Gesammtgefässsystem hätten ausüben können, wie psychische Erregungen, irgend welche Unlustgefühle u. dergl., ausgeschaltet worden. Unter diesen Umständen darf angenommen werden, dass Blutdruckschwankungen, wie sie an den Armgefässen zu registriren sind, nicht allein in diesen selbst, sondern gleichzeitig auch in anderen Gefässgebieten ausgelöst werden.

Erst wenn mehrfache, an verschiedenen Tagen vorgenommene Messungen bei den abgesonderten, zu Bett gehaltenen Versuchspersonen annähernd constante Blutdruckwerthe aufwiesen, wurde vor Beginn der eigentlichen Versuche an einem Tage in Abständen von 10-30 Minuten 4-5-6 Stunden, also meist den ganzen Vormittag hindurch, der systolische und diastolische Druck stets zwei- bis dreimal hintereinander gemessen, damit auf diese Weise eine Curve für die physiologischen Blutdruckschwankungen zu Stande kam. An den Rauchtagen selbst wurde erst dann mit dem Rauchen begonnen, nachdem an zwei oder drei verschiedenen Zeitpunkten die Ausgangswerthe des Blutdrucks festgelegt waren.

Die Werthe für den systolischen (s) und diastolischen Druck (d) sowie die Grösse der Amplitude (A) und der Blutdruckquotient (Q), endlich die Pulsfrequenz (P) wurden in Form von Curven registrirt, und oberhalb dieser Curven noch die bei den einzelnen Messungen beobachtete Grösse der Schwankungen der Manometernadel eingezeichnet.

Ich habe zwar darauf verzichtet, die mit sämmtlichen Versuchspersonen angestellten Versuche hier wiederzugeben, habe aber noch immerhin grössere Versuchsreihen herausgegriffen, da es mir darauf ankam, in möglichst umfassender Weise den Effect des in verschiedener Form und in verschiedener Intensität betriebenen Tabackrauchens auf das Gefässsystem klarzulegen.

1. Paul A., 34 Jahr. Wegen Spitzenaffection und leichter vasomotorischer Störungen in Behandlung. Im Uebrigen ohne positiven Organbefund. Kein Alkohol-, kein Nicotinabusus.

Die fortlaufende Messung am 5. 6. 07 (Tafel XIV, Curve I, 1) ergiebt Schwankungen für s um $24 \mathrm{~cm} \mathrm{H} \mathrm{H}_{2} \mathrm{O}$, für d um $14 \mathrm{~cm} \mathrm{H}_{2} \mathrm{O}$. Wenn man von der ersten Messung absieht, betragen die Schwankungen für s nur $12 \mathrm{om}_{2} \mathrm{O}$.

Das Rauchen von 2 Wendt-Cigarren am 6. 6. 07 (Tafel XIV, Curve I, 2) lässt keine nennenswerthen Blutdruckschwankungen zu Stande kommen.

Sobald aber am 24.6. 2 mittelschwere Cigarren gerauoht werden (Tafel XIV, Curve I, 3) steigt s noch während des Rauchens um $14 \mathrm{~cm}$ und $\mathrm{d}$ um $18 \mathrm{~cm} \mathrm{H}_{2} \mathrm{O}$ an. $1 / 2$ Stunde nach beendetem Rauchen ist $s$ wieder auf den Ausgangswerth abgesunken, d dagegen um weitere $8 \mathrm{~cm}, 1-2$ Stunden später sogar um $12 \mathrm{~cm}$ angestiegen, so dass zu dieser Zeit $\mathrm{A}$ ganz erbeblich, und zwar bis um $28 \mathrm{~cm} \mathrm{H}_{2} \mathrm{O}$, verkleinert ist. Infolgedessen auch $Q$ von 0,44 auf 0,26 abgesunken, und $P$ von 84 auf $60-72$.

2. Gustar v. R., $24 \mathrm{Jahr}$, Arbeiter. Nie krank gewesen. Am 11.6. 07 Aufnahme ins Krankenhaus wegen Erysipels. Vom 21. 6. ab fieberfrei. Hat bisher nur mässig geraucht. 
Die fortlaufende Messung am 26. 6. (Tafel XIV, Curve II, 1) lässt im Verhalten von $s$ und $d$ sowie $A$ und $Q$ nur geringfügige Schwankungen orkennen.

Am 30. 6. 07 rauchte $R$. innerbalb einer Stunde 2 Wendt-Cigarren (Tafel XIV, Curve II, 2), wobei s vorübergehend während des Rauchens um $8 \mathrm{~cm} \mathrm{H}_{2} \mathrm{O}$ ansteigt, etwa 10 Minuten nach beendetem Rauchen um $10 \mathrm{~cm}$ abgesunken ist, um dann bald wieder den Anfangswerth zu erreichen. d allmählich im Ganzen $10 \mathrm{~cm}$ unter den Ausgangswerth absinkend. P zwischen 84 und 72.

Am 9. 7. wurden in der Zeit ron 24 Stunden 4 Wendt-Cigarren geraucht (Tafel XIV, Curve II, 3). Dabei macht sich nach $1 / 2$ Stunde ein Ansteigen von $\mathrm{s}$ um $10 \mathrm{~cm}$ bemerkbar. Gegen Schluss des Rauchens s wieder auf den Ausgangswerth und 1 Stunde später $8 \mathrm{~cm}$ unter denselben abgesunken. d die ganze Zeit über so gut wie unverändert. A infolge des schliesslichen Absinkens von s gegenüber dem Ausgangswerth $10 \mathrm{~cm}$ verkleinert.

Beim Rauchen von 2 mittelschweren Cigarren am 2. 7. ('Tafel XIV, Curve II, 4) und 3 Cigarren der gleichen Sorte am 6. 7. (Tafel XIV, Curve II, 5) ist s nur ganz unbeträchtlich, d dagegen $12-14 \mathrm{~cm} \mathrm{H}_{2} \mathrm{O}$ über den Ausgangswerth erhöht, und zwar am ausgesprochensten 1 Stunde nach Boginn des Rauchens. Zu dieser Zeit $\mathrm{A}$ daher verkleinert. P ohne nennenswerthe Schwankungen.

3. Simon P., 37 Jahr, Ziegeleiarbeiter. Vom 25. 8.-17. 9. wegen rheumatischer Beschwerden ohne objectiven Befund auf Station gelegen. Körperorgane ohne krankhaften Befund. Bis $3 / 4$ Liter Schnaps pro die.

Die fortlaufende Messung am 9. 9. 07 (Tafel XIV, Curve III, 1) lässt erkennen, dass sich sowohl s wie d, dementsprechend auch $A$ und $Q$ kaum nennenswerth verändert.

Das Rauchen von 2 Wendt-Cigarren am 10.9. (Tafel XIV, Curve III, 2) bewirkt zunächst noch während des Rauchens ein leichtes Ansteigen von s um $10 \mathrm{~cm} \mathrm{H}_{2} \mathrm{O}$, während d erst nach boendetem Rauchen vorübergehend ansteigt. Im Ganzen sind jedenfalls die Blutdruckschwankungen keine erheblichen.

Am 13. und 14. 9. rauchte P. je 2 Stück mittelschwere Cigarren, wobei eine typische Beeinflussung des Blutdruckes beobachtet werden konnte, und zwar am 13.9. (Tafel XIV, Curve III, 3) ein wechselndes Steigen und Fallen von s zwischen dem Ausgangswerth ron $140 \mathrm{~cm}$ und $156 \mathrm{~cm} \mathrm{H}_{2} \mathrm{O}$, noch während des Rauchens Ansteigen von $\mathrm{d}$ um 12, nach beendetem Rauchen bis auf $20 \mathrm{~cm} \mathrm{H}_{2} \mathrm{O}$ über den Ausgangswerth. Also wechselnde Verkleinerung von $A$ und $Q$. P obne wesentliche Aenderung. Am 14. 9. (Tafel XIV, Curve III, 4) steigt $s$ noch wäbrend des Rauchens von 140 auf $168 \mathrm{~cm} \mathrm{H}_{2} \mathrm{O}$ und $\mathrm{d}$ von 76 auf $112 \mathrm{~cm} \mathrm{H}_{2} \mathrm{O}$ an. Nach beendetem Rauchen fallen beide Werthe wieder, bleiben aber noch wesentlich über den Ausgangsdruck erhöht. Infolge des ziemlich gleichzeitigen Ansteigens und Wiederabsinkens von $s$ und $d$ ist A dem Ausgangswerth gegenüber nicht erheblich verkleinert. $P$ schwankender als Tags zuvor.

4. Iudwig S., $40 \mathrm{Jahr}$, Hochofenarbeiter. Kam wegen rheumatischer Beschwerden ins Krankenhaus. Ohne objectiven Befund. Zeitweise Alkoholabusus. Bis vor $1 / 4$ Jahr starker Raucher.

Bei der fortlaufenden Messung am 28.10.07 (Tafel XV, Curve IV, 1) ergeben sich leichte Schwankungen für s zwischen 138 und $127 \mathrm{~cm} \mathrm{H}_{2} \mathrm{O}$. $d$ fast constant. Ebenso $\mathrm{P}$, zwischen 84 und 88 .

Das Rauchen von 2 Wendt-Cigarren am 29. 10. (Tafel XV, Curve IV, 2) bzw. zwei Stück Dr. Wimmer's Patenteigarren am 30. 10. (Tafel XV, Curve IV, 3) verursacht etwas deutlichere Blutdruckschwankungen als am Tage der fortlaufenden Messung. Besonders $d$ weist $1 / 2$ Stunde nach Beginn des Rauchens einen kurzdauernden Anstieg von 12 resp. $16 \mathrm{~cm} \mathrm{H}_{2} \mathrm{O}$ auf, so dass um diese Zeit eine geringfügige Verkleinerung von $A$ und $Q$ bemerkbar ist. $P$ in dem ersten Falle von 96 auf 84, im 
zweiten Falle vorübergehend von 88 auf 72 abgesunken. Im Grossen und Ganzen handelte es sich nicht um erhebliche Blutdruckschwankungen.

Am 31.10. (Tafel XV, Curve IV, 4) und 8.11. (Tafel XV, Curve IV, 5) rauchte $S$. je 2 mittelschwere Cigarren. An beiden Tagen ein deutliches Ansteigen von d, und zwar am 1. Versuchstage schon während des Rauchens um über $20 \mathrm{~cm} \mathrm{H} \mathrm{H}_{2} \mathrm{O}$, am 2. Versuchstage erst 1 Stunde nach beendetem Rauchen ausgesprochen. A dementsprechend ganz besonders am 31. 10. beträchtlich verkleinert, am hochgradigsten $1 / 4-11 / 2$ Stunden nach beendetem Rauchen. $P$ von 90 resp. 96 auf 80 absinkend.

Beim Rauchen von 2 Importen am 7. 11. (Tafel XV, Curve IV, 6) kommt es noch während des Rauchens durch Sinken von $\mathrm{s}$ um $10 \mathrm{~cm} \mathrm{H}_{2} \mathrm{O}$ und leichtes Ansteigen von $d$ zu einer Verkleinerung von A. $1 / 2$ Stunde nach beendetem Rauchen ist $d \mathrm{um}$ 10 und $11 / 2$ Stunden um $16 \mathrm{~cm} \mathrm{H}_{2} \mathrm{O}$ angestiegen, so dass $\mathrm{A}$ und auch $\mathrm{Q}$ bis etwas unter den Ausgangsdruck abgesunkenem s ganz erheblich verkleinert sind. Schwankungen von $\mathrm{P}$ ausgesprochener als an den anderen Rahetagen.

Das Rauchen von 10 Stück leichten russischen Cigaretten am 5. 11. (T'afel XV, Curve IV, 7 ) löst keine erheblichen Blutdruckschwankungen aus. s jedenfalls kaum verändert, d lediglich wäbrend des Rauchens für $1 / 2$ Stunde um $10 \mathrm{~cm} \mathrm{H}_{2} \mathrm{O}$ angestiegen. A daher ohne erbebliche Schwankungen, ebenso P.

Das Rauchen von 8 Stück schwereren russischen Cigaretten am 6.11. (Tafel XV, Curve IV, 8) lässt noch während des Rauchens ein Ansteigen von $s$ um 10 und $d$ um $16 \mathrm{~cm} \mathrm{H}_{2} \mathrm{O}$ erkennen. Sclswankungen von $\mathrm{A}$ geringfügig, $\mathrm{P}$ zwischen 96 und 78 .

5. Albert N., Eisenarbeiter, 42 Jahre. Wegen rechtsseitiger Radialislähmung in Behandlung. Verdacht auf Arteriosklerose. Sonst ohne nachweisbare Organveränderungen. $1 / 4-1 / 2$ Liter Schnaps und $11 / 2$ Liter Bier pro die. Täglich einige Cigarren.

Tafel XV, Curve V, 1 bringt die Werthe für die fortlaufende Messung, welche für s Schwankungen um 8, für d um 15, für $\mathrm{A}$ um $20 \mathrm{~cm} \mathrm{H}_{2} \mathrm{O}$ und für $\mathrm{Q}$ um 0,12 ergiebt. $\mathrm{P}$ zwischen 60 und 72.

Beim Rauchen von zwei Wendt-Cigarren am 10.9. 07 (Tafel XV, Curve V, 2) lassen sich keine nennenswerthen Blutdruckschwankungen constatiren.

Dagegen bat das Rauchen von zwei mittelschweren Cigarren am 13. 9.07 (Tafel XV, Curve V, 3) etwas dentlichere Bintdruckschwankungen zur Folge. Noch während des Rauchens Ansteigen von $s$ bis auf $18 \mathrm{~cm}$ und von $d$ bis auf $20 \mathrm{~cm} \mathrm{H}_{2} \mathrm{O}$ über den Ausgangsdruck, wobei A und $Q$ so gut wie unverändert bleiben. 40 Minuten nach beendetem Rauchen beginnen s und $d$ wieder bis zum Ausgangswerth abzusinken. $\mathrm{P}$ zwischen 60 und 72. Bei dem am 14.9. wiederholten Rauchen von zwei Cigarren (Tafel XV, Curve V, 4) sind ähnliche Blutdruckschwankungen zu constatiren. Noch während des Rauchens Ansteigen von s und d um $10 \mathrm{~cm} \mathrm{H}_{2} 0,1 / 2-1^{1} / 2$ Stunden nach beendetem Rauchen ist $\mathrm{s}$ um $10 \mathrm{~cm} \mathrm{H}_{2} \mathrm{O}$ unter den Ausgangswerth abgesunken, d zwar gleichfalls vorübergehend auf den Ausgangswerth zurückgekehrt, meist aber $10 \mathrm{~cm} \mathrm{H}_{2} \mathrm{O}$ über diesen erhöht, so dass $\mathrm{A}$ zeitweise um $16 \mathrm{~cm} \mathrm{H}_{2} \mathrm{O}$ verkleinert ist.

Am 23.9. 07 rauchte N. in der Zeit von 2 Stunden 40 Minuten vier Stück Dr. Wimmer's nicotinarme Cigarren (Tafel XV, Curve V, 5), wobei s in den ersten zwei Stunden sich nur unwesentlich änderte, dann allmällich um $10 \mathrm{~cm} \mathrm{H}_{2} \mathrm{O}$ absank, während d nur geringfügige Schwankungen aufwies. Beim Rauchen von vier Stück WendtCigarren am 24. 9. (Tafel XV, Curve V, 6) kommt es bei fast unverändertem s zu einem Ansteigen von d, eine Stunde nach begonnenem Rauchen bis auf $14 \mathrm{~cm} \mathrm{H}_{2} \mathrm{O}$ über dem Ausgangswerth.

Um festzustellen, in welcher Weise der Blutdruck bei N. durch das Rauchen mehrerer Cigarren im Laufe des Tages beeinflusst werde, wurden am 30.9. 07 fortlaufende Messungen des Blutdruckes im Sitzen vorgenommen. Auf Tafel XV, Curve V, 7 lassen sich ziemlich beträchtliche Blutdruckschwankungen für $\mathrm{s}$ und $\mathrm{d}$, meist in 
gleichem Sinne, erkennen, so dass A keine erheblichen Ånderungen erfährt. Am 2. 10.07 rauchte N. sieben Cigarren (Tafel XV, Curve V, 8). Hierbei zeigte s keine nennenswerthen Schwankungen, während d zeitweise deutlich erhöht war, so dass sich $\mathrm{A}$ bis zu $20 \mathrm{~cm} \mathrm{H}_{2} \mathrm{O}$ verringerte. $\mathrm{P}$ zwischen 96 and 72 .

6. Heinrich S., 23 jähriger Molkereigehilfe. Seit dem 18. Jahre Epileptiker. Wegen eines Anfalles ins Krankenhaus gebracht, woselbst er vom 24. 8.-24. 10, verblieb, ohne dass während dieser Zeit ein Anfall beobachtet werden konnte. Keine nachweisbaren Organveränderungen, in Sonderheit Herz und Gefässsystem ohne nachweisbare krankhafte Veränderungen. Mässig im Trinken und Rauchen.

Die am 18.9.07 vorgenommene fortlaufende Messung (Tafel XVI, Curre VI, 1) ergiebt für die Werthe $\mathrm{s}, \mathrm{d}, \mathrm{P}, \mathrm{A}, \mathrm{Q}$ fast gerade, fortlaufende Linien, d. h. geringfügige Schwankungen.

Beim Rauchen von zwei Stück Dr. Wimmer's nicotinarmen Cigarren am 23. 9. (Tafel XVI, Curve VI, 2), sowie von zwei Wendt-Cigarren am 24. 9. (Tafel XVI, Curve VI, 3) sind nur geringfügige Schwankungen der einzelnen Blutdruckwerthe zu bemerken, beispielsweise etwa 10 Minuten nach beendetem Rauchen der Wendt-Cigarren ein Ansteigen von a um $10 \mathrm{~cm} \mathrm{H}_{2} \mathrm{O}$.

Das Rauchen von zwei mittelschweren Cigarren dagegen am 25. 9. (Tafel XVI, Curve VI, 4) hat deutliche Blutdruckschwankungen zur Folge. Schon wälhrend des Rauchens Ansteigen von d um $12 \mathrm{~cm} \mathrm{H}_{2} \mathrm{O}$. Eine Stunde später, kurz vor Beendigung des Rauchens, ist auch $\mathrm{s}$ um $10 \mathrm{~cm} \mathrm{H}_{2} \mathrm{O}$ angestiegen, um bald nachher $\mathrm{um} 10 \mathrm{~cm}$ unter dem Ausgangsdruck abzusinken, während d meist $12 \mathrm{~cm} \mathrm{H}_{2} \mathrm{O}$ über den Ausgangsdruck erhöht bleibt, so dass $\mathrm{A}$ um $14-22 \mathrm{~cm} \mathrm{H}_{2} \mathrm{O}$ verkleinert ist. P zwischen 76 und 88 .

Am 26. 9. 07 rauchte S. in der Zeit von zwei Stunden zehn leichtere russische Cigaretten (Tafel XVI, Curve VI, 5). $11 / 2$ Stunden nach begonnenem Rauchen ist $\mathrm{s}$ um $10 \mathrm{~cm} \mathrm{H}_{2} \mathrm{O}, 3 / 4-1 / 2$ Stunde nach beendetem Rauchen um weitere $10 \mathrm{~cm} \mathrm{H}_{2} \mathrm{O}$ abgesunken, d nur leichteren Schwankungen im Sinne eines geringfügigen Absinkens bis um $10 \mathrm{~cm} \mathrm{H}_{2} \mathrm{O}$ unterworfen, so dass $\mathrm{A}$ sich zeitwoise um $14 \mathrm{~cm} \mathrm{H}_{2} \mathrm{O}$ verlileinert, während $Q$ unwesentliche Schwankungen aufweist. $P$ zwischen 80 und 92.

Das Rauchen von zehn Stück schwereren russischen Cigaretten (Tafel XVI, Curve VI, 6) hat nach $1-1 \frac{1}{2}$ Stunden ein Sinken von $\mathrm{s}$ um $10 \mathrm{~cm} \mathrm{H}_{2} \mathrm{O}$ und ein Ansteigen von d um $12 \mathrm{~cm} \mathrm{H}_{2} \mathrm{O}$ zur Folge. A um $12 \mathrm{~cm}$ verkleinert. $1 / 2-1$ Stunde nach beendetem Rauchen ist $\mathrm{s}$ um weitere $10 \mathrm{~cm} \mathrm{H}_{2} \mathrm{O}$ gesunken, $\mathrm{d} 5-10 \mathrm{~cm}$ über den Ausgangsdruck erhöht, A also zeitweise um $24 \mathrm{~cm}$ verkleinert. Auch $Q$ zeigt diesmal Schwankungen zwischen 0,25 und 0,4 . P während des Rauchens von 72 auf 84 angestiegen, nach beendetem Rauchen zwischen 60 und 70 hin- und herschwankend.

Aus den vorliegenden Messungsergebnissen, die der besseren Uebersichtlichkeit wegen in Form von Curven veranschaulicht sind, geht zunächst mit aller Deutlichkeit hervor, dass beim Rauchen nicotinhaltiger Cigarren der Blutdruck in ganz bestimmter Weise beeinflusst wird. Dass freilich die Blutdruckschwankungen bei den verschiedenen Versuchspersonen sich nicht immer in genau der gleichen Weise vollziehen, ist wohl selbstverständlich. Denn abgesehen von der individuell verschiedenen Toleranz gegen Nicotin dürfte ein und dasselbe Agens das Gefässsystem verschiedener Personen bezw. ein und derselben Person zu verschiedenen Zeiten ganz verschieden stark beeinflussen, je nach der Anspruchsfähigkeit der Gefässe im Einzelfalle oder im gegebenen Augenblick. Individuen mit leicht erregbaren $V$ asomotoren werden weit ausgesprochener reagiren, sobald sie zwei Cigarren rauchen, als Andere mit weniger lcbhaftem Spiel 
der Vasomotoren. Bei einem Vasomotoriker sehen wir also die Nicotinwirkung sozusagen unter dem Vergrösserungsglas der gesteigerten Reactionsfähigkeit seiner Gefässe. Daher möchte ich auch die bei dem Vasomotoriker A. beim Rauchen zweier mittelschwerer Cigarren zu beobachtenden Schwankungen des Blutdrucks zum Ausgangspunkt der Besprechungen machen.

Noch während des $1 \frac{3}{4}$ Stunden anhaltenden Rauchens der zwei Cigarren kommt es zu einem Ansteigen des systolischen Druckes bis um $14 \mathrm{~cm}$ und des diastolischen bis um $18 \mathrm{~cm} \mathrm{H}_{2} \mathrm{O}$. Die Amplitude verändert sich also während dieser Zeit nicht wesentlich. Nach beendetem Rauchen dagegen steigt der diastolische Druck weiter um $8 \mathrm{~cm}$ $\mathrm{H}_{2} \mathrm{O}$ an, während der systolische wieder bis auf den Ausgangswerth abfällt. Die Amplitude erfährt jetzt also eine erhebliche Verkleinerung, und zwar deshalb, weil der diastolische Druck im Ganzen um $26 \mathrm{~cm}$ $\mathrm{H}_{2} \mathrm{O}$ über den Ausgangsdruck erhöht ist. Der Effect des Rauchens auf das Gefässsystem macht sich somit nicht bloss während der Rauchprocedur selbst, sondern in verstärktem Maasse bis $2^{1} / 2$ Stunden nach Beendigung derselben geltend. Zu der Zeit der stärksten Einwirkung ist die Pulsfrequenz von 78 auf $60-64$ Schläge herabgesetzt.

Das Wesentliche der durch das Tabackrauchen hervorgerufenen Gefässwirkung ist demnach das Ansteigen des diastolischen Druckes, das auch in allen anderen Versuchen stets zu constatiren ist. Allerdings beträgt der Anstieg zuweilen nur $10 \mathrm{~cm} \mathrm{H}_{2} \mathrm{O}$ und ist nicht immer schon während des Rauchens, stets aber $1-1 \frac{1}{2}$ Stunden nach beendeter Rauchprocedur vorhanden. Aenderungen des systolischen Druckes sind nicht so regelmässig zu verzeichnen. Bei Versuchspersonen 2 und 4 bleiben sie beispielsweise vollständig aus. In Fall 3 dagegen erfolgt ein vorübergehender Anstieg des systolischen Druckes um $28 \mathrm{~cm} \mathrm{H}_{2} \mathrm{O}$ gegen Ende der Rauchprocedur und in Fall 5 in dem einen Versuch um 14, in dem anderen um $10 \mathrm{~cm} \mathrm{H}_{2} \mathrm{O}$. Von typischen Aenderungen der Pulsfrequenz, etwa im Sinne einer Verlangsamung wie bei dem Vasomotoriker A., kann in den übrigen Versuchen nicht die Rede sein. Uebrigens als Beleg dafür, dass das Gefässsystem ein und derselben Versuchsperson zu verschicdenen Zeiten durch das gleiche Agens nicht immer in gleicher Weise beeinflusst wird, darf der 49 jährige M. S. gelten, der an drei verschiedenen Tagen zwei Cigarren rauchte, wobei der diastolische Druck das erste Mal um 28, das zweite und dritte Mal nur um $14 \mathrm{~cm} \mathrm{H}_{2} \mathrm{O}$ anstieg.

Was das Rauchen von zehn Stück russischen Cigaretten (einer leichteren und einer etwas schwereren Sorte) anlangt, so machen sich dabei ähnliche Blutdruckänderungen wie beim Rauchen von zwei Cigarren bemerkbar, und $\mathrm{zwar}$ ist die Reaction auf die schwerere Sorte eine intensivere. Im Allgemeinen lässt sich sagen, dass zehn Stück leichte russische Cigaretten das Gefässsystem etwas geringfügiger als zwei Cigarren und Letztere wieder geringfügiger als zehn Stück schwerere russische Cigaretten beeinflussen. Demnach bedeutet das Rauchen von zehn Stück schwereren russischen Cigaretten eine grössere Schädigung für das Gefässsystem als das Rauchen zweier mittelschweren Cigarren. 
Interessant ist es nun, zu verfolgen, welche geringfügige Blutdruckänderungen beim Rauchen nicotinarmer Cigarren zu bemerken sind. Bei dem Vasomotoriker A. beispielsweise bewegen sich die Schwankungen beim Rauchen von 2 Wendt-Cigarren in derselben Breite wie bei der fortlaufenden Messung. In Fall 2 kommt es wohl beim Rauchen von 2 resp. 4 Wendt-Cigarren zu geringfügigen Schwankungen des systolischen Druckes, der diastolische bleibt aber so gut wie unverändert, in Fall 3 verhält sich der Blutdruck beim Rauchen von 2 Wendt-Cigarren kaum anders als bei der fortlaufenden Messung, ebenso in den folgenden Fällen 4 bis 6 . Alle diese Versuche beweisen mit genügender Deutlichkeit, dass nicotinarme Cigarren, und zwar gleichviel, ob solche der Firma Wendt oder der Marke Navahoe, das Gefässsystem entschieden weniger alteriren als die gewöhnlichen nicotinhaltigen Cigarren oder Cigaretten. In einem Falle von Mitralsténose, der hier nicht mit aufgeführt ist, zeigte sich ganz besonders eclatant die unverkennbar ungünstige Beeinflussung des Allgemeinbefindens durch eine mittelschwere Cigarre gegenüber der völligen Unschädlichkeit von 2 Stück Wendt-Cigarren. Während beim Rauchen einer mittelschweren Cigarre sich deutlich Intoxicationssymptome einstellten (Schweissausbruch, Uebelkeit, Erbrechen, Herzklopfen), wurden dio beiden Wendt-Cigarren anstandslos vertragen. Das Verhalten des Blutdruckes liess sich hierbei leider nicht mit wünschenswerther Genauigkeit verfolgen, da wegen der stark irregulären und inäqualen Pulse eine Bestimmung des Minimaldruckes nicht möglich war.

Da die meisten Raucher täglich nicht nur 2, sondern 5-10 Cigarren und darüber bzw. 25-30 Cigaretten beanspruchen, so stellte ich noch bei den beiden folgenden Versuchspersonen fest, in welcher Weise der Blutdruck beeinflusst wird, wenn Tags über 8-10 Cigarren (mittelschwere bzw. nicotinarme) oder 25-30 Stück russische Cigaretten geraucht werden. Bei diesen Rauchversuchen wurden die Versuchspersonen nicht zu Bett gehalten, sondern konnten, wie im täglichen Leben auch, umhergehen und sich nach Belieben beschäftigen. Im übrigen waren sie selbstverständlich vor den eigentlichen Versuchen so häufig gemessen worden, dass die einzelnen Messungen ihre Aufmerksamkeit in keiner Weise mehr erregten.

\section{Versuche.}

7. Wilhelm L., 27 jähriger gesunder Arbeiter; wegen einer Armfractur im Krankenhaus.

Die fortlaufende Messung am 10.6. (Tafel XVI, Carve VII, 1) ergiebt wenigstens am Vormittag ziemlich constante Werthe für $s$, ganz besonders aber für d. Erst am Nachmittag $\mathrm{s} \mathrm{um} 18 \mathrm{~cm} \mathrm{H}_{2} \mathrm{O}$ angestiegen und $\mathrm{A}$ in Folge dessen entsprechend vergrössert.

Am 11. 6. rauchte L. 10 Stück nicotinarme Cigarren "Navahoe" (Tafel XVI, Curve VII, 2) schon nach der ersten Cigarre s und d um $30 \mathrm{bzw} .20 \mathrm{~cm} \mathrm{H}_{2} \mathrm{O}$ angestiegen; nach Beendigung der vierten wieder je $10 \mathrm{~cm} \mathrm{H}_{2} \mathrm{O}$ abgefallen. Nach der Mittagspause beim Beginn der fünften Cigarre $s$ und d wieder am Ausgangswerth, in der Folgezeit bis um $24 \mathrm{~cm} \mathrm{H}_{2} \mathrm{O}$ ansteigend. $\Lambda$ zeigt Schwankungen bis um $14 \mathrm{~cm} \mathrm{H}_{2} \mathrm{O}$, $\mathrm{P}$ zwischen 60 and 76.

Beim Rauchen von 10 Stück Wendt-Cigarren am 15.6. (Tafel XVI, Curve VII, 3) fallen die Schwankungen ähnlich, aber geringfügiger aus; jedenfalls $A$ und $Q$ fast constant. 
Das Rauchen von 10 Stück mittelschwerer Cigarren am 12.6.08 (Tafel XVI, Curve VII, 4) verursacht allmähliches Ansteigen von s und d. Bei der zehnten Cigarre ist $\mathrm{s}$ um 30 , d $10 \mathrm{~cm} \mathrm{H} \mathrm{H}_{2} \mathrm{O}$ über den Ausgangswerth erhöht. Nach beendetem Rauchen fällt $\mathrm{s}$ allmählich um 20 , d dagegen steigt weiter um $10 \mathrm{~cm} \mathrm{H}_{2} \mathrm{O}$ an. A weist ziemlich beträchtliche Schwankungen auf, zwischen 78 und $50 \mathrm{~cm} \mathrm{H}_{2} \mathrm{O}, \mathrm{P}$ zwischen 70 und 88 .

30 Stïck leichte russische Cigaretten, die L. am 13. 6. rauchte (Taf $\theta$ XVI, Curve VII, 5); haben etwa denselben Effect wie 10 Cigarren. Nur macht sich gleich zu Anfang, 2 Stunden andauernd, ein besonders stark ausgesprochenes Ansteigen von s und d von 28 bzw. $22 \mathrm{~cm} \mathrm{H}_{2} \mathrm{O}$ bemerkbar. Am Nachmittag ist $\mathrm{s}$ und meist nur $10 \mathrm{om} \mathrm{H}_{2} \mathrm{O}$ höher als zu Beginn. Nach beendetem Rauchen steigt $d$ allmählich um weitere $10 \mathrm{~cm} \mathrm{H} \mathrm{H}_{2} \mathrm{O}$ an, während s bis $6 \mathrm{~cm}$ unter den Ausgangswerth absinkt, so dass A um diese Zeit erheblich verkleinert ist.

8. Max G., 28jähriger gesunder Arbeiter; wegen leiohter Armverletzung im Krankenhans.

Die sogenannten physiologischen, täglichen Blutdruckschwankungen fallen am 10.6. nicht sebr beträchtlich aus (Tafel XVII, Curve VIII, 1). Abgesehen von den beiden ersten, niedrigen Werthen für $s$ und $d$ sind für $s$ Schwankungen zwischen 154 und 160 , also um fast $6 \mathrm{~cm} \mathrm{H}_{2} \mathrm{O}$ und für $\mathrm{d}$ zwischen 96 und 104, also um $8 \mathrm{~cm} \mathrm{H}_{2} \mathrm{O} \mathrm{zu}$ verzeichnen.

Am 11. 6. rauchte G. 10 Stück nicotinarme Cigarren "Navahoe" (Tafel XVII, Curve VIII, 2). Hierbei zeigt d nur geringfügige Schwankungen um $10 \mathrm{~cm} \mathrm{H}_{2} \mathrm{O}$. s dagegen schwankt bei $150 \mathrm{~cm}$ Ausgangsdruck zwischem 136 und $168 \mathrm{~cm} \mathrm{H}_{2} \mathrm{O}$, in Folge dessen auch grössere Schwankungen von $A$. P unverändert.

10 Stück Wendt-Cigarren, die G. am 5. 6. rauchte (Tafel XVII, Curve VIII, 3) lassen keine deutlichen Schwankungen der einzelnen Blutdruckwerthe erkennen. Die Curve verhält sich kaum anders als die der fortlaufenden Messung rom 10.6.

Deutliche Blutdruckschwankungen treten zu Tage beim Rauchen von 8 Stück mittelschwerer Cigarren am 12.6 (Tafel XVII, Curve VIII, 4), indem etwa 2-3 Stunden nach beendetem Rauchen $s$ nach vorübergebendem Anstieg bis zum Ausgangswerth und darunter, d um 10-18 $\mathrm{cm} \mathrm{H}_{2} \mathrm{O}$ über seinen Ausgangswerth erhöht ist. A ist zeit= weise also ziemlich beträchtlich verkleinert, schwankt zwischen 78 und $46 \mathrm{~cm} \mathrm{H}_{2} 0$. $Q$ zwischen 0,3 und 0,46. P zwischen 90 und 76 .

Eine bemerkenswerthe Beeinflussung des Blutdruckes ist ferner beim Rauchen von 20-25 Stück leichter russischer Cigaretten zu constatiren (Tafel XVII, Curve VIII, 5). d während des Rauchens fast constant $10 \mathrm{~cm}$ über den Ausgangspunkt erhöht, $s$ bald bis $18 \mathrm{~cm}$ über den Ausgangswerth, bald bis $4 \mathrm{~cm}$ unter den Ausgangswerth, A zeitweise deutlich verkleinert. 2-4 Stunden nach beendetem Rauchen s 16 und d 20 bis 24 om $\mathrm{H}_{2} \mathrm{O}$ über den Ausgangsdruck orhöht. Alles in allem Blutdruckschwankungen, wie sie weder an dem Tage, wo nicht geraucht, noch an dem Tage, wo nicotinarme Cigarren geraucht wurden, beobachtet werden konnten.

Die zuletzt angefübrten Versuche ergeben ohne Weiteres, dass 8 bis 10 Cigarren, die im Laufe eines Tages geraucht werden, das Gefässsystem in unzweideutiger Weise alteriren. Der systolische Druck unterliegt häufigeren, im allgemeinen regellosen Schwankungen und zeigt dabei eine steigende Tendenz. Der diastolische Druck wird gleichfalls erböht, im ersten Falle bis um 24, im anderen bis um $16 \mathrm{~cm} \mathrm{H}_{2} \mathrm{O}$. Etwa 2 bis 3 Stunden nach beendetem Rauchen convergiren die beiden Werthe, so dass zu dieser Zeit die Amplitude eine deutliche Verkleinerung erfährt. Die Beeinflussung des Herzgefässsystems durch Tabackrauchen 
markirt sich auch durch deutliche, wenngleich nicht gesetzmässige Schwankungen der Pulsfrequenz. Während die Pulscurve an den Vorversuehstagen fast in gerader Linie verläuft, weist sie bei L. Schwankungen zwischen 66 und 88 Schlägen auf, bei G. dagegen ein allmähliches Absinken um 10-20 Schläge unter den Ausgangswerth.

25-30 Stück leichte russische Cigaretten - von passionirten Cigarettenrauchern dürtten häufig genug weit mehr am Tage geraucht werden - lassen ähnliche Blutdruckschwankungen zu Stande kommen wie 8-10 mittelschwere Cigarren. Der systolische Druck ist zeitweise bis um 30, der diastolische in beiden Fällen bis auf $20-24 \mathrm{~cm} \mathrm{H}_{2} \mathrm{O}$ über den Ausgangswerth erhöht. Nach beendetem Rauchen in den späten Nachmittagsstunden convergiren beide Werthe, so dass die Amplitude eine Verkleinerung erfährt.

Bezüglich der nicotinarmen Cigarren muss gesagt werden, dass diese selbst in Mengen von 10 Stück das Gefässsystem jedenfalls sehr viel weniger in Mitleidensehaft ziehen als nicotinhaltige Cigarren. Ganz besonders beim Rauchen der Wendt-Cigarren bleiben bei beiden Versuchspersonen nennenswerthe Blutdruckschwankungen aus, während beim Rauchen der nicotinarmen Cigarren "Navahoe" sich Blutdruckänderungen bemerkbar machen. Allerdings verlaufen dieselben stets gleichsinnig. Ein Convergiren der Werthe für den systolischen und diastolischen Druck findet nicht statt, demgemäss auch keine Verkleinerung der Amplitude, wie dies beim Rauchen der nicotinhaltigen Cigarren der Fall ist. Wenn nur 2 oder 4 Stück nicotinarme Cigarren, gleichviel welcher Sorte, geraucht wurden, war ein derartiger Unterschied in der Wirkung nicht vorhanden. Allerdings hatte man da eine Einwirkung auf das Gefässsystem überhaupt nicht erkennen können.

Die deutliche Beeinflussung des Kreislaufes durch das Rauchen nicotinhaltiger Cigarren und Cigarretten einerseits, die geringfügigen Veränderungen des Blutdruckes beim Rauchen nicotinarmer Cigarren andererseits drängen schon von selbst zu der Annahme, dass der Nicotingehalt ides Tabackrauches die charakteristische Gefässwirkung bedingt. Und $n$ der That erweisen sich nach den eingehenden Untersuchungen Lehmann's und seiner Schüler ausser dem Nicotin sämmtliche Bestandtheile des Tabackrauches, wie Kohlenoxyd, Schwefelwasserstoff, Blausäure, Pyridin und seine Homologen, als unschädlich.

Aus der sehr umfassenden Arbeit Lehmann's und seiner Schüler seien einige Bemerkungen über das Rauchen und die beim Rauchen aufgenommenen Nicotinmengen hervorgehoben: Beim Rauchen entsteht ein Neben- und ein Hauptstrom; Letzterer gelangt in die Mundhöhle, wo etwa $44,7-48,5 \mathrm{pCt}$. des in ihm enthaltenen Nicotins, bei schweren Cigarren 51-70 pCt. absorbirt werden, während beim Cigarettenrauchen nur $25-30 \mathrm{pCt}$. des Hauptstromes zur Absorption gelangen. Der Hauptstrom ist um so grösser, je hastiger und intensiver geraucht wird, bzw. je kürzer die Pausen zwischen den einzelnen Zügen ausfallen. Demgemäss ist auch die Menge des absorbirten Nicotins eine schwankende. Nach Sinnhold beträgt der Nicotingehalt von 20 in Europa fabricirten Cigarren 1,009-2,9 pCt., von Cigarettentaback 0,8-2,887, von Pfeifen- 
taback $0,518-0,771 \mathrm{pCt}$, von nicotinfreien Cigarren "Navahoe" 0,8 bis 0,5 bis 0,4 pCt. Von dem Gesammtnicotingehalt leichter Cigarren werden im Mittel etwa $11 \mathrm{pCt}$., bei starken Cigarren etwa $17 \mathrm{pCt}$. und bei Cigaretten $10 \mathrm{pCt}$. aufgenommen, d. h. aus einer mittleren Cigarre 5 bis $10 \mathrm{mg}$ und aus einer guten Cigarette $1,2-2,3 \mathrm{mg}$.

Bezüglich der Giftigkeit des Nicotins führt Lehmann aus der Toxikulogie von Kunkel an, dass $20 \mathrm{mg}$ Nicotin schon eine gefährliche Dosis darstellt, wenn sie resorbirt werden, und in Selbstversuchen verschiedener Schüler Lehmann's, die sämmtlich regelmässige, zum Theil sehr starke Raucher waren, lösten $15 \mathrm{mg}$ Nicotin schon deutliche Intoxikationssymptome aus. Für die Thatsache, dass Cigarren von gleichem Nicotingehalt nichtsdestoweniger verschieden stark wirken können, giebt Lehmann drei Erklärungsmöglichkeiten:

1. Könnte aus den starken Cigarren das Nicotin vollständiger in den Rauch übergehen als aus den schwachen, von gleichem Nicotingehalt, d. h. in den schwachen reichlicher verbrennen.

2. Es könnte die in den Hauptstrom eingehende Menge verschieden gross sein. Gehen das eine Mal 30, das andere Mal 60 pCt. in den Nebenstrom, so muss das von fundamentaler Bedeutung sein.

3. Es könnten aus der gleichen im Rauch vorhandenen Nicotinmenge verschiedene Quantitäten absorbirt werden. Da reine Nicotindämpfe von der Mundhöhle besser absorbirt werden als rauchbaltige, so müssen wir annehmen, dass bei "leichten" Cigarren mit hohem Nicotingehalt die Absorption des Nicotins durch gewisse Rauchbestandtheile besonders stark herabgesetzt werden kann.

Besonders die zweite der drei Möglichkeiten dürfte die schwache Wirkung nicotinarmer Cigarren ins rechte Licht setzen. Denn ihr geringerer Nicotingehalt allein, der von anderen Cigarren nur um das Doppelte oder Dreifache übertroffen wird, kann ihre jedenfalls mehr als 2-3 Mal geringere Schädlichkeit nicht hinreichend erklären. Die zu meinen Versuchen verwandten nicotinarmen Cigarren "Navahoe" und der Firma Wendt brannten ausserordentlich leicht, so dass wohl ein grosser Nebenstrom entstand und von dem Gesammtnicotingehalt procentualiter weniger Nicotin absorbirt wurde als dies bei den nicht so leicht verbrennenden nicotinhaltigen Cigarren der Fall ist. Auch Ratner weist in seinen experimentellen Untersuchungen „über die physiologische Wirkung des Tabackrauches auf den Organismus" auf die relative Unschädlichkeit der nicotinarmen Cigarren hin. „Bei Controlversuchen mit sogenanntem nicotinfreiem Taback bleiben die Kreislaufstörungen mehr oder weniger ganz aus oder sind nur in geringerem Maasse vorhanden."

Da sich die Rauch- resp. Nicotinwirkung stets durch ein Ansteigen des diastolischen Druckes documentirt, so dürfen wir annehmen, dass die Gefässe unter dem Einfluss des Tabackrauchens in einen erhöhten Contractionszustand gerathen. Ihr Tonus wird pathologisch verstärkt. Dass dem wirklich so ist, lässt sich durch Versuche beweisen, wie ich 
sie seiner Zeit schon zur Erklärung der Gefässwirkung des Alkohols herangezogen hatte und auf die ich hier deshalb nur kurz eingehen kann.

Das Eintauchen des Armes in Wasser von $41-42^{\circ} \mathrm{C}$. lührt zu einer kaum nennenswerthen Verkleinerung der Amplitude durch geringfügiges Absinken des systolischen und event. auch des diastolischen Druckes, das Eintauchen in kaltes Wasser von $10^{\circ} \mathrm{C}$. dagegen zu einer deutlichen Vergrösserung der Amplitude infolge eines ziemlich beträchtlichen Ansteigens des systolischen, bei einer geringfügigen Erhöhung des diastolischen Druckes. Durch den Kältereiz wird der Gefässtonus erhöht (Ansteigen des diastolischen Druckes), deswegen selbst bei gleichbleibendem Füllungszuwachs der Armgefässe Vergrösserung der Amplitude.

Wie bei den Alkoholversuchen bestimmte ich ausser der Reactionsfähigkeit noch im Stadium der Nicotinwirkung die Pulsfrequenz in Bettlage (A) und nachher in aufrechter Haltung (D), und zwar um festzustellen, ob unter dem Einfluss von Nicotin beim Lagewechsel ebenso erhebliche Differenzen in der Steigerung der Pulsfrequenz resultiren wie unter dem Einfluss von Alkohol.

In den folgenden Curven ist der Zeitpunkt, wo der Uebergang von $A$ in D erfolgte, durch ein D unterhalb der Pulslinie vermerkt, während' der Zeitpunkt des Eintauchens in warmes oder kaltes Wasser durch einen Pfeil mit darunterstehendem $\frac{\mathrm{E}}{\mathrm{k} . \mathrm{W}}$. oder $\frac{\mathrm{E}}{\mathrm{W} . \mathrm{W} .}$ gekennzeichnet ist.

\section{Versuche.}

9. Karl Sch., 51 Jahre, Bergmann. Wegen linksseitiger Omalgie in Behandlung. Ohne irgend welchen Organbefund. Täglich $1 / 4-1 / 2$ Liter Schnaps.

Bemerkenswerth auf Curve IX, 1, Tafel XVIII ist einmal eine kaum nennenswerthe Aenderung von $\mathrm{P}$ bei D. Die Reaction auf Eintauchen in warmes Wasser besteht in einem Abfallen von $\mathrm{s}$ um 8 und d um $4 \mathrm{~cm} \mathrm{H}_{2} \mathrm{O}$, also in einer Verkleinerung von $\mathrm{A}$ um $4 \mathrm{~cm} \mathrm{H}_{2} \mathrm{O}$. Beim Eintauchen in kaltes Wasser in einem Ansteigen von s bis um 30 , von d bis um $6 \mathrm{~cm} \mathrm{H}_{2} \mathrm{O}$, also Vergrösserung von A um $24 \mathrm{~cm} \mathrm{H}_{2} \mathrm{O}$. Hervorgehoben zu werden verdient der Umstand, dass gleichzeitig mit der Erhöhung des Gefässtonus durch den Kältereiz ein deutliches Grösserwerden der Oscillationen am Tonometer, z. B. auf 5 bis 6 Theilstriche, zu constatiren war.

Am 25. 9. rauchte Sch. zwei mittelschwere Cigarren (Tafel XVIII, Curve IX, 2). Was zunächst den Einfluss des Rauchens auf den Blutdruck anbelangt, so ist bereits eine Stunde nach Beginn desselben ein Anstieg von $\mathrm{s}$ um 14, von d um $10 \mathrm{~cm} \mathrm{H}_{2} \mathrm{O} \mathrm{zu}$ constatiren. Kurz vor Beendigung der zweiten Cigarre und noch $11 / 4$ Stunde nachher hält sich s $8-10 \mathrm{~cm}$, d $16 \mathrm{~cm} \mathrm{H}_{2} \mathrm{O}$ über dem Ausgangswerth, sodass A um $8 \mathrm{~cm}$ kleiner wird. Von den Gefässreactionen auf Eintauchen in warmes resp. kaltes Wasser noch während des Rauchens ist zu sagen: Das warme Wasser lässt die Gefässe unbeeinflusst. Nach Eintauchen in kaltes Wasser steigt $\mathrm{s}$ bis um 40 , d um $10 \mathrm{~cm} \mathrm{H} \mathrm{H}_{2} \mathrm{O}$ über den Ausgangswerth. A also um $30 \mathrm{~cm} \mathrm{H}_{2} \mathrm{O}$ vergrössert. Oscillationen deutlich grössere. 11/2 Stunde nach beendetern Rauchen Wärmeapplication ohne Effect, während unter Einwirkung des kalten Wassers s um 34, d um $10 \mathrm{~cm} \mathrm{H}_{2} \mathrm{O}$, A also um $24 \mathrm{~cm} \mathrm{H}_{2} \mathrm{O}$ ansteigt. Noch $4 \frac{1}{2}$ Stunde nach beendetem Rauchen erhöht sich s' nach Kälteapplication um 32, d um 4, A demnach um $28 \mathrm{~cm} \mathrm{H}_{2} \mathrm{O}$. $\mathrm{P}$ ohne wesentliche Schwankungen, auch nicht beim Lagewechsel (D).

Nach einer Kälteapplication fällt also der Anstieg von s und A entschieden beträchtlicher aus, sobald das Gefässsystem der Einwirkung des Tabackrauchens aus- 
gesetzt jst. Ganz besonders schön und prägnant tritt in folgendem Fall zu Tage, wie verschieden der Effect der Kälteapplication sich gestalten kann, einmal wenn der Kreislauf nicht beeinflusst und dann, wenn nur zwei Cigarren geraucht worden sind.

10. Oscar M., 41 Jahre, Arbeiter. Wegen rheumatischer Beschwerden in Behandlung, ohne nachweisbare Organerkrankungen. Vor 10 Jahren rechtsseitige Hemiplegie, die sich aber vollständig zurückgebildet hat. Lues negirt. Wassermann'scbe Reaction negativ. Früher $1 / 2$ Liter Schnaps und etwa 3 Liter Bier pro die, seit dem Schlaganfall angeblich etwas weniger Alkohol.

Zu Curve X, 1, Tafel XVIII, auf welcher gelegentlich der fortlaufenden Messung die auf Eintauchen in kaltes und warmes Wasser erfolgenden Blutdruckschwankungen registrirt sind, ist weiter nichts zu bemerken, als dass letztere in diesem Falle weniger ausgiebig ausfallen wie im vorigen. Die Anspruchsfähigkeit des Gefässsystems bezw. einzelner Gefäss $\theta$ auf thermische Reize bewegt sich offenbar hier in engeren Grenzen.

Ueber die Einwirkung von drei Cigarren am 30. 9. (Tafel XVIII, Curve X, 2) ist zu sagen, dass schon nach der ersten Cigarre s um 14, d um $10 \mathrm{~cm} \mathrm{H}_{2} \mathrm{O}$ erhöht ist, $s$ dann weiter nicht mehr ansteigt, d dagegen kurz vor Beendigung des Ranchens im Ganzen um $22 \mathrm{~cm} \mathrm{H} \mathrm{H}_{2} \mathrm{O}$ erhöht, $\mathrm{A}$ also um $16 \mathrm{~cm} \mathrm{H} \mathrm{H}_{2} \mathrm{O}$ verkleinert ist. Noch $2 \frac{1}{2}$ Stunden nach beendetem Rauchen, wo $\mathrm{P}$ von 88 auf 72 vermindert ist, behält $d$ den erhöhten Werth bei. Das Eintauchen des Armes in kaltes Wasser, zum ersten Male während des Rauchens, bedingt ein Ansteigen von $\mathrm{s}$ um $16 \mathrm{~cm} \mathrm{H}_{2} \mathrm{O}$ bei unverändertem d, zum zweiten Male eine Stunde nach beendetem Rauchen ein Ansteigen von s um $14 \mathrm{~cm} \mathrm{H}_{2} \mathrm{O}$ bei gleichfalls verändertem $\mathrm{d}$. Wärmeapplication ohne nennenswerthen Effect.

Das Ansteigen von s und A zu einer Zeit, wo der Kältereiz die Gefässe, die unter Nicotinwirkung stehen, trifft, ist in diesem Falle zwar geringfügiger als im vorhergehenden, aber deutlich wahrnehmbar, zumal es ausserbalb der Nicotinwirkung fast ganz vermisst wird.

Wie die eben erwähnten Versuche ergeben, reagiren die Gefässe in der That unter dem Einfluss von Nicotin in einer Weise, die nach den vorausgegangenen Erörterungen die Existenz einer erhöhten Gefässspannung beweist. Und dabei gilt noch zu berücksichtigen, dass in dem einen Falle beim Rauchen von zwei Cigarren das Ansteigen des diastolischen Druckes, also die Erhöhung des Gefässtonus, sich lange nicht so erheblich gestaltet hatte als in vielen anderen Fällen. Gleichwohl grosse Amplitude bei Einwirkung des Kältereizes, eben weil eine doch in erhöhtem Spannungszustande befindliche Gefässwand getroffen wurde. In dem anderen Falle hatte der Kältereiz an sich keine nennenswerthen Schwankungen der Amplitude, also auch keine ersichtliche Erhöhung des Gefässtonus zur Folge gehabt. Trotz dieser geringfügigen Anspruchsfähigkeit der Gefässwand kam im Stadium der Nicotinwirkung durch den Kältereiz eine erhebliche Vergrösserung der Amplitude zu Stande, ein deutliches Anzeichen dafür, dass jetzt der Gefässtonus merklich erhöht war.

Das beim Rauchen in den Organismus aufgenommene Nicotin wirkt also zweifellos auf die Gefässe ein, und zwar versetzt es nicht bloss die Gefässwand in einen erhöhten Spannungszustand, sondern verursacht auch Veränderungen in der Gefässwand selbst. Denn im Thierexperiment lassen sich rach den Untersuchungen von Adler und Häusel, Baylac, A mouroux und Gouget sowohl durch intravenöse Injection von Nicotin 
wie durch stomachale Einverleibung von Tabackinfus an den Gefässen Erkrankungsprocesse erzeugen, welche den nach Adrenalininjectionen auftretenden Veränderungen sehr ähnlich sind. Boveri nimmt an, dass die Herde primär in der Media entstehen, in Folge einer Combination der druckerhöhenden mit der toxischen Wirkung des Nicotins und hält den Process für analog der menschlichen Arteriosklerose.

Experimentelle Forschung und klinische Erfahrung befinden sich jedenfalls in seltener Uebereinstimmung darüber, dass dem Nicotin eine nicht zu unterschätzende Bedeutung in der Aetiologie von Gefässerkrankungen zuzuschreiben ist. Nach Erb ist der Taback „von der hervorragendsten Bedeutung für das Entstehen des intermittirenden Hinkens, d. h. der vorwiegend in den Unterschenkeln localisirten Arteriosklerose". In zwei Beobachtungsreihen von 35 und 38 derartig Erkrankten konnte er 9- bzw. 14 mal ausser Tabacksmissbrauch keine sonstigen Schädlichkeiten nachweisen. Schlesinger fand unter 46 Männern mit intermittirendem Hinken 31 sehr starke Raucher, und sah mehrere Male nach Aussetzen des Rauchens deutliche Besserung des Leidens. Auch die dem intermittirenden Hinken entsprechenden pathologischen Vorgänge, wie sie sich an anderen Organen (Darm, Herz, Gehirn) bei zeitweiligen Contractionszuständen erkrankter Gefässe (Sklerose der Abdominal-, Coronar-, Cerebralgefässe) abspielen, also ganz allgemein für die Dyspragia angiosclerotica intermittens, dürfte der Tabackabusus in ätiologischer Beziehung eine grössere Rolle spielen, als bisher von vielen Seiten zugestanden wird. Es unterliegt für mich gar keinem $Z$ weifel, dass bei schon bestehender Coronarsklerose beispielsweise ein Anfall von Angina pectoris durch Rauchen ausgelöst werden kann. So hatte ich Gelegenheit, bei einem älteren an Coronarsklerose erkrankten Herrn, bei welchem anamnestisch weder Excesse in baccho et venere noch eine luetische Infection (Wassermann negativ), sondern lediglich starkes Rauchen in Betracht kam, direct zu beobachten, wie das Uebertreten des Rauchverbotes sofort anginoide Beschwerden provocirte. Durch das Rauchen waren die ohnehin erkrankten Coronargefässe in eine Art Krampfzustand versetzt worden. In Folge dessen vorübergehende schlechtere Durchblutung des Herzmuskels, die ja immer als Vorbedingung für das Zustandekommen eines stenokardischen Anfalles zu gelten hat. Dass so manche Menschen bis in ihr hohes Alter hinein $6-8$, ja $15-20$ Cigarren im 'Tag ungestraft rauchen können, beweist nichts für die Unschädlichkeit des Rauchens, sondern bestätigt höchstens wieder einmal die Thatsache, dass einzelne Individuen eine ganz ausserordentliche Resistenz gegenüber gewissen Giften besitzen.

\section{Zusammenfassung.}

Das Rauchen von 2 mittelschweren Cigarren bedingt in typischen Fällen ganz charakteristische Blutdruckschwankungen, und zwar kommt es noch während des Rauchens zu einem deutlichen Ansteigen des diastolischen Druckes, indess der systolische Druck den Ausgangswerth wenig oder gar nicht überschreitet, eher etwas darunter absinkt. Diese Blut- 
Beeinflussung des systolischen u. diastolischen Blutdrucks durch Tabackrauchen. 365

druckveränderungen sind oft noch 2 Stunden nach beendetem Rauchen deutlich ausgesprochen.

Das Wesentliche der Nicotingefässwirkung beruht also darauf, dass ein Contractionsreiz auf die Gefässwand ausgeübt wird, wodurch diese einen erhöhten Tonus annimmt. Diese Tonussteigerung kann durch Prüfung der Anspruchsfähigkeit der Gefässe im Stadium der Nicotinwirkung direct erwiesen werden.

Die Pulsfrequenz ist nicht immer in gleicher Weise beeinflusst. Zuweilen erweist sie sich als verlangsamt; meist ist sie lediglich grösseren Schwankungen unterworfen als in der Norm.

Auf 8-10 Stück russische Cigaretten reagirt das Gefässsystem ähnlich wie auf 2 mittelsehwere Cigarren. Zwischen leichterer und schwerer Sorte ist ein deutlicher Unterschied in der Wirkung zu erkennen.

2-4 Stück nicotinarme Cigarren der Firma Wendt bzw. der Marke "Navahoe" beeinflussen den Blutdruck nicht in erkennbarer Weise.

Grössere Mengen, 8-10 Stück Cigarren, über den Tag vertheilt, sind nicht gleichgiltig für das Verhalten des Blutdruckes, wenn auch die vielen blutdruckherabsetzenden und blutdrucksteigernden Einflüsse des täglichen Lebens gesetzmässige Schwankungen nicht zu Stande kommen lassen. Gegenüber den nicotinhaltigen sind die nicotinarmen Cigarren von entschieden geringem Effect, während 25-30 Stück Cigaretten sich in ihrer Wirkung kaum von 8-10 nicotinhaltigen Cigarren unterscheiden.

Die Messungsergebnisse, welche eine deutliche Beeinflussung der Gefässe durch das Tabackrauchen als sicher erwiesen haben, bestätigen hier die auch sonst experimentell erwiesene Möglichkeit, dass Nicotin Gefässveränderungen im Sinne einer Arteriosklerose hervorzurufen vermag.

Bei allen Zuständen, die dem Krankheitsbilde der Dyspragia angiosclerotica intermittens zuzurechnen sind, ist absolute Enthaltsamkeit vom Rauchen unbedingt geboten, da die gefässverengernde Wirkung des Nicotins den Zustand verschlechtern bzw. direct einen Anfall hervorrufen kann.

\section{Literatur.}

1) Erb, Klinische Beiträge zur Pathologie des "intermittirenden Hinkens". Münchener med. Wochenschr., 1910. No. 21, 22. - Zur Klinik des intermittirenden Hinkens. Ebenda. 1910. No. 47 und 1911. No. 47.

2) John, Ueber die Beeinflussung des systolischen und diastolischen Blutdrucks durch Genuss alkoholischer Getränke verschiedener Concentration. Diese Zeitschrift. Bd. 5. 
$366 \mathrm{~J} \mathrm{ohn,} \mathrm{Beeinflussung} \mathrm{d.} \mathrm{systolisch.} \mathrm{u.} \mathrm{diastolisch.} \mathrm{Blutdrucks} \mathrm{durch} \mathrm{Tabackrauchen.}$

3) Lehmann, Vorläufige Mittheilungen über Tabackstudien. Hygien. Rundschau. 1907. No. 18.

4) Jehmann und seine Mitarbeiter, Chemische und toxikologisohe Studien über Taback, Tabackrauch und das Tabackrauchen. Arch. f. Hyg. Bd. 68.

5) Lohde, Ueber chronische Tabackvergiftung. Inaug.-Diss. 1902.

6) Nicolai und Staehelin, Ueber die Einwirkung des Tabaokgenusses auf die Circulationsapparate. Diese Zeitschr. Bd. 8.

7) Ratner, Experimentelle Untersuchungen über die physiologische Wirkung des Tabackrauches auf den Organismus. Arch. f. d. ges. Physiol. Bd. 113.

8) Saltykow, Die experimentell erzeugten Arterienveränderungen in ibrer Beziehung zu Arteriosklerose und verwandten Krankheiten der Menschen. Centralbl. f. allg. Pathol, u. pathol. Anat. Bd. 19. No. 8,9.

9) Schlesinger, Zur Klinik des intermittirenden Hinkens. Münchener med. Wochenschr. 1910. No. 50. 

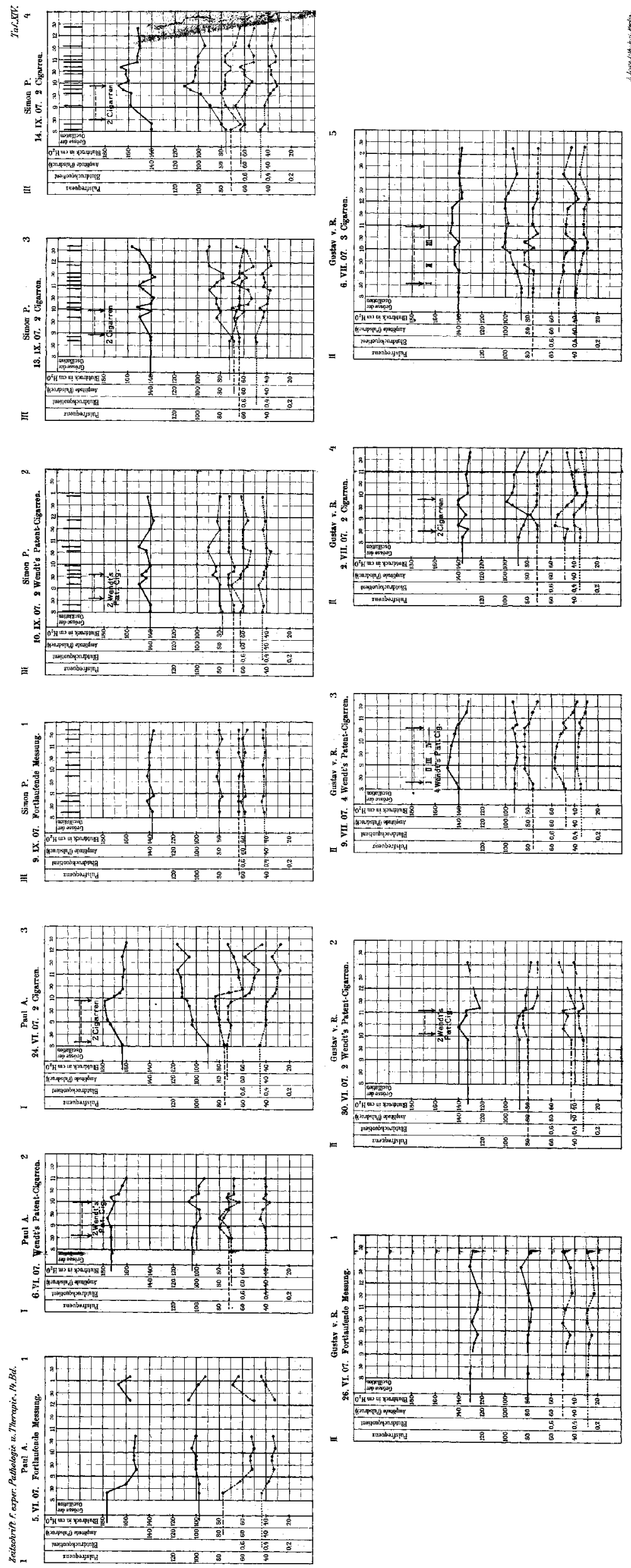

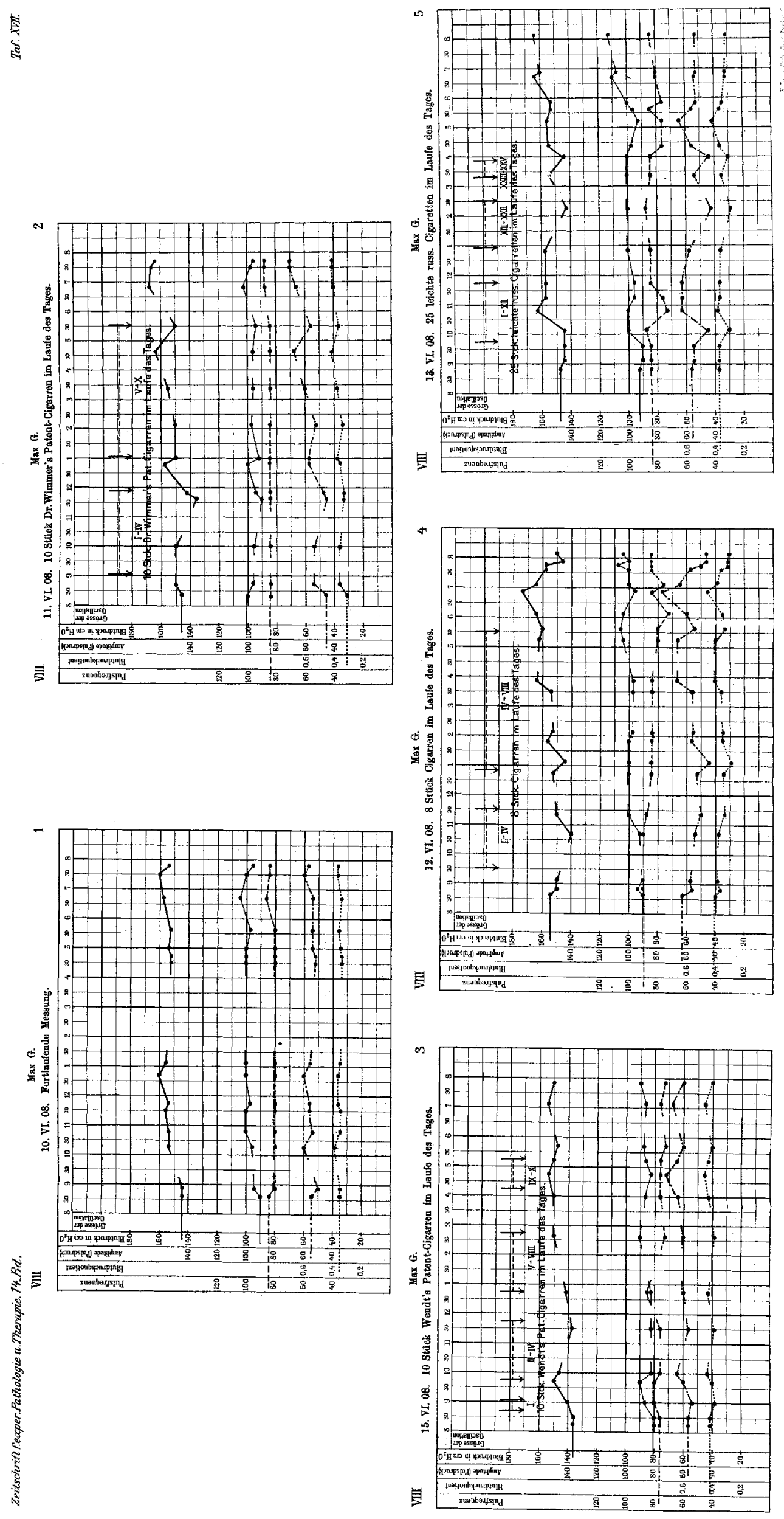

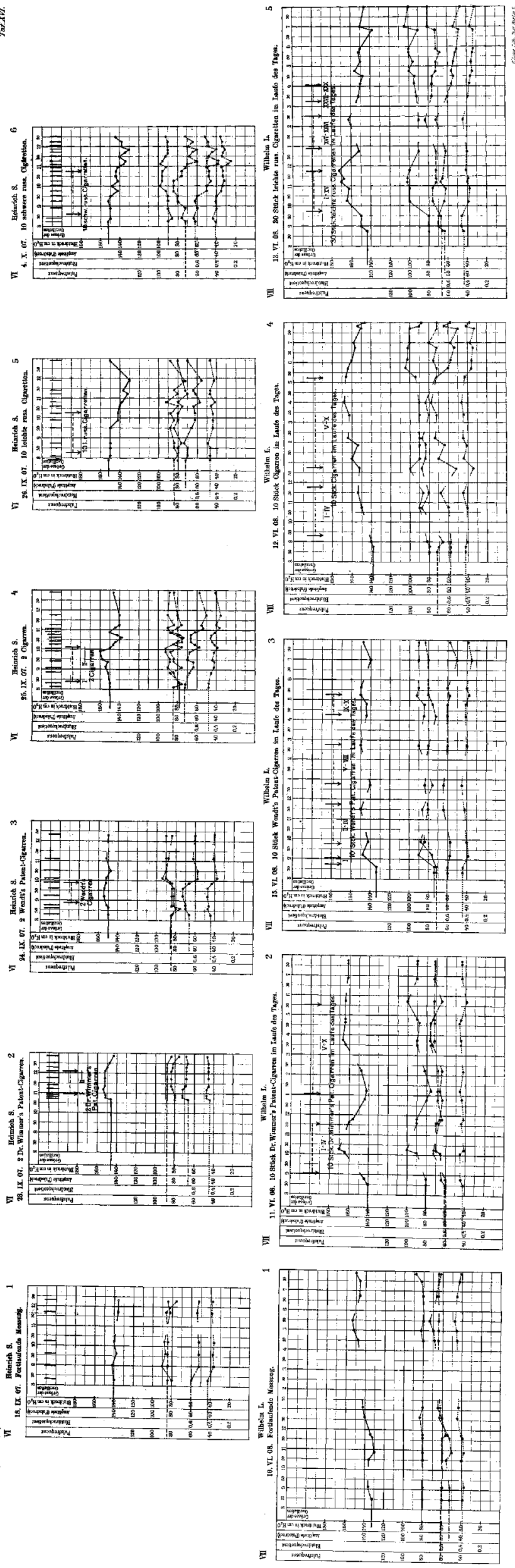

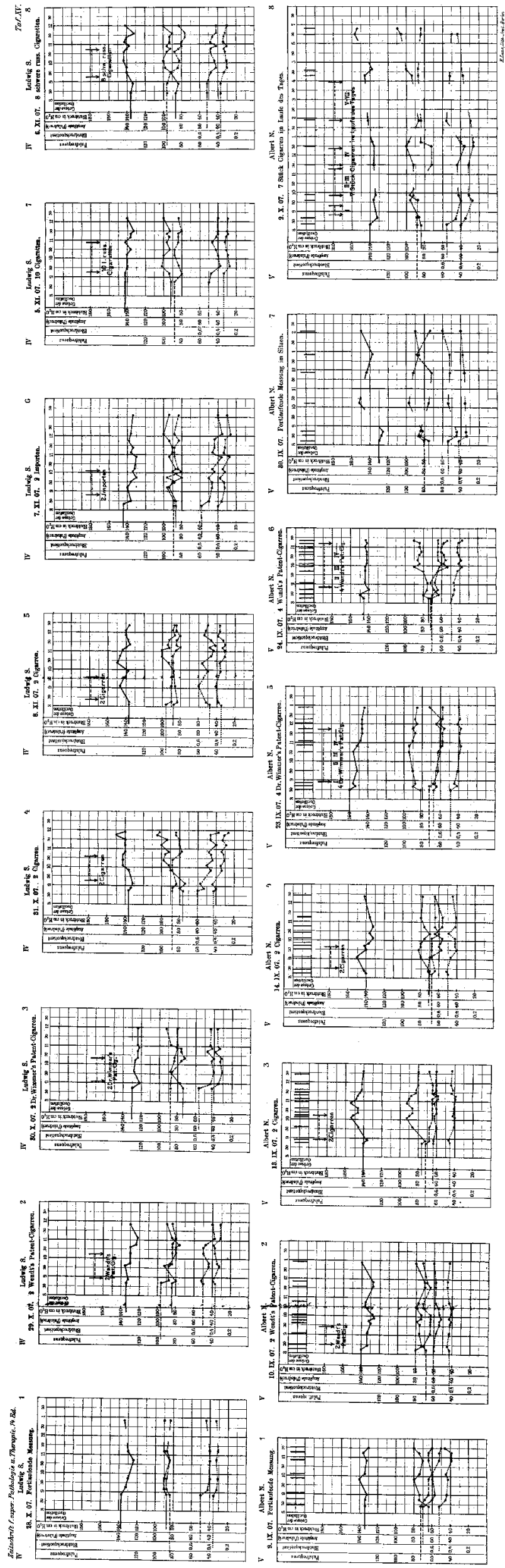

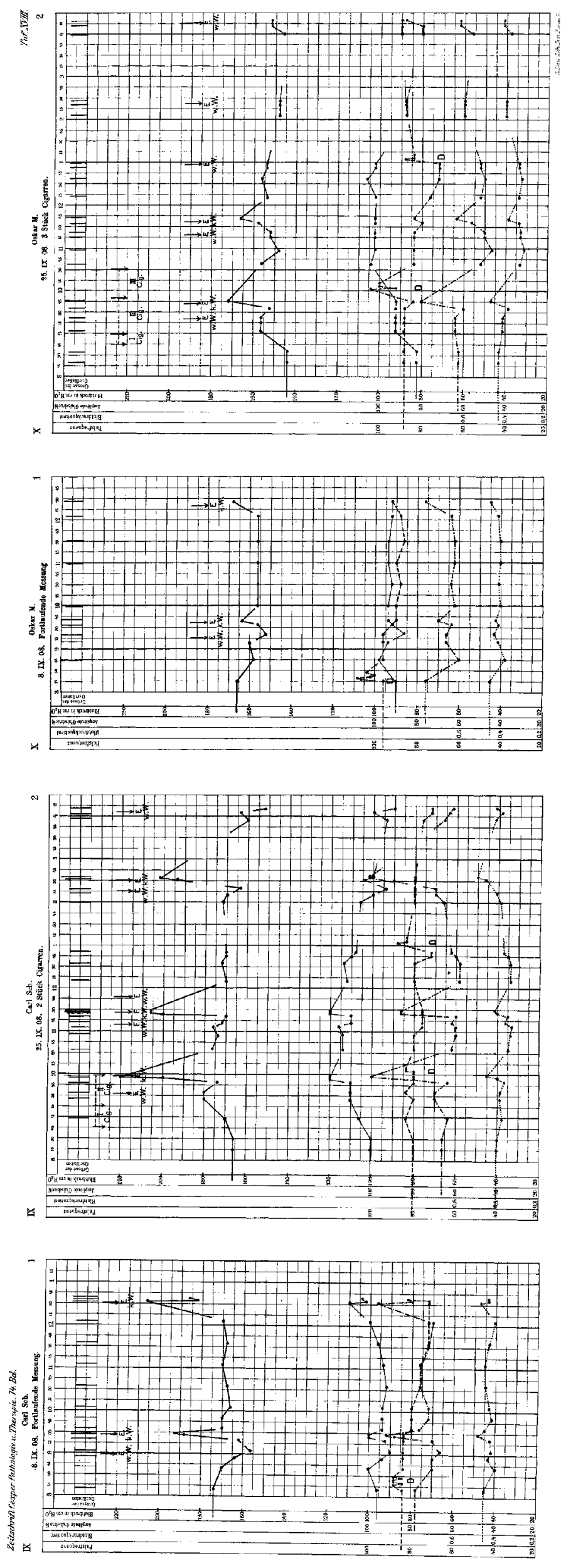